\title{
Dual coronary embolization associated with atrial fibrillation: a case report
}

\author{
Yonggang YUAN, Zesheng XU* \\ Department of cardiology, Cangzhou Central Hospital, No. 16 Xinhua Road, Cangzhou 061000, Hebei, China \\ *Correspondence: xuzeshengc@sina.com \\ https://doi.org/10.37175/stemedicine.v2i8.99
}

\begin{abstract}
Background: Thrombotic occlusion of the coronary artery, which succeeds the atherosclerotic plaque rupture or erosion, gives rise to a major portion of acute myocardial infarction (AMI) incidences. Nevertheless, coronary embolism is gaining increasing recognition as another important factor contributing to AMI.
\end{abstract}

Case presentation: A 72-year-old woman with atrial fibrillation (AF) and diabetes mellitus histories, presented with chest pain radiating to the left arm and shoulder that began 6 hours prior to admission. Electrocardiogram revealed AF plus ST-segment elevation in the anterior leads.

Intervention: Patient was first treated with anti-platelet agents (aspirin plus ticagrelor) and atorvastatin. Emergency coronary angiography depicted multi-site coronary embolization of the left circumflex artery $(L C X)$ and the left anterior descending artery (LAD). Blood flow was not restored after intracoronary injection of $600 \mathrm{ug}$ tirofiban. $40 \mathrm{mg}$ recombinant human prourokinase was then administered via aspiration thrombectomy catheter.

Outcome: Two weeks later, coronary angiography showed no residual obstructive lesion in the LCX and LAD with TIMI (thrombolysis in myocardial infarction) 3 flow.

Conclusion: Primary percutaneous coronary intervention is the most effective measure. In the case of failed blood flow restoration, thrombolytic treatment in both intravenous and intracoronary route should be considered.

Keywords: Embolization · Acute myocardial infarction · Atrial fibrillation · Thrombolytic treatment

\section{Introduction}

The characteristics of acute myocardial infarction (AMI) include atherosclerotic plaque rupture, fissure, ulceration, dissection or erosion with subsequent thrombus in coronary arteries in most cases, which leads to a disturbed balance between the demand and supply of myocardial oxygen and subsequent myocardial necrosis (1).

Received: Aug 25, 2021; Accepted: Sep 9, 2021.

(c) The Author(s). 2021 This is an Open Access article distributed under the terms of the Creative Commons License (http://creativecommons.org/licenses/by/4.0/) which permits unrestricted use, distribution, and reproduction in any medium or format, provided the original work is properly cited.
Clinically, myocardial ischemia can often be identified based on the medical history of the patient as well as the electrocardiography. Symptoms of ischemia include upper extremity, chest, epigastric or mandibular pain or discomfort (at rest with exertion), or ischemic equivalents such as dyspnea or fatigue, which may last about $20 \mathrm{~min}$. In most cases, the AMI-associated discomfort is diffuse - not positional, nor localized, and not altered by local movements - and it is sometimes accompanied by nausea, syncope, and diaphoresis. Those symptoms, however, are not myocardial ischemia specific, and therefore may be mistakenly diagnosed as gastrointestinal, pulmonary, musculoskeletal, or neurological disorders. Further, it is 
possible that MI occurs with only atypical symptoms (eg. cardiac arrest or palpitations), or no symptom at all; for instance in female patients, diabetics, post-operative or critically ill patients, or the elderly (2). Cautious assessment of such patients is encouraged, particularly with a falling and/or rising trend of cardiac biomarkers.

Meanwhile, coronary artery embolism (CE) is regarded as a significant non-atherosclerotic factor contributing to AMI. However, its prevalence is still not clear due to the difficulty to generate acute diagnosis (3). Atrial fibrillation (AF) is the leading cause of CE, and correlates with high risks of thromboembolic incidence. The AF prevalence is predicted to rise due to population aging (4). However, the clinical characteristics, incidence rate, and prognosis of AF remain largely elusive.

We hereby described a case study of a 72 year old female who was admitted to our hospital with persistent AF and ST elevation myocardial infarction (STEMI).

\section{Case description}

This study was performed in June 2020 in Department of cardiology, Cangzhou Central Hospital. A 72-yearold female with histories of complete atrioventricular block, persistent AF, and diabetes mellitus, presented with complaints regarding chest pain radiating to the left arm and shoulder that began 6 hours prior to her arrival. Electrocardiogram revealed AF, complete atrioventricular block and ST-segment elevation in the anterior leads. 10 minutes after first medical contact she was given a combination of antiplatelet agents (aspirin, ticagrelor, $\mathrm{IIb} / \mathrm{III}$ a receptor inhibitors) and atorvastatin. Emergency coronary angiography demonstrated multi-site coronary embolization, with abrupt 'cut-off' at the proximal of the left anterior descending artery (LAD) and the distal end of the left circumflex artery (LCX). No significant obstructive lesion was found in the right coronary artery (Figure 1).

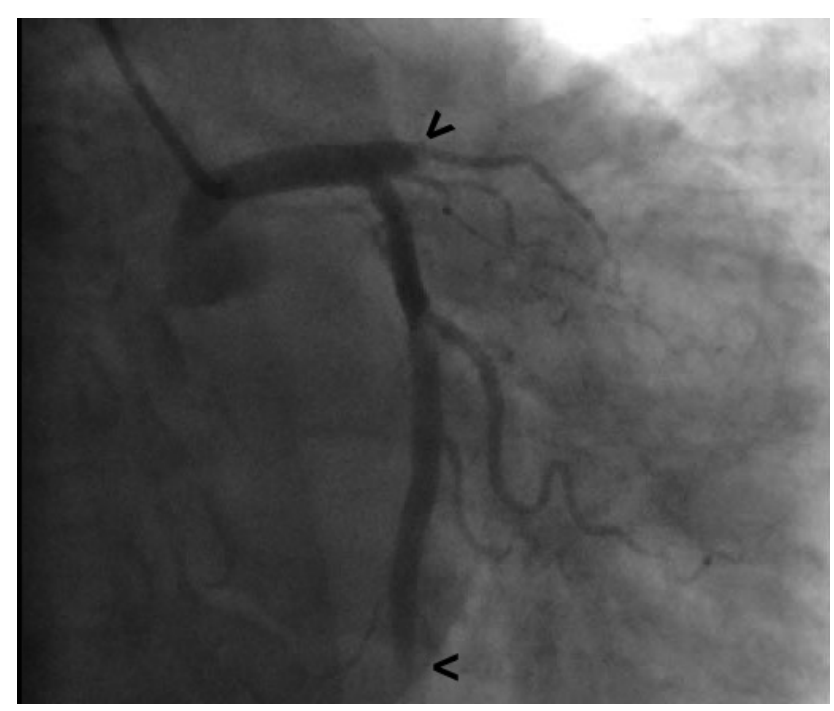

Figure 1. Emergency coronary angiography, arrow heads indicate abrupt 'cut-off' sites.

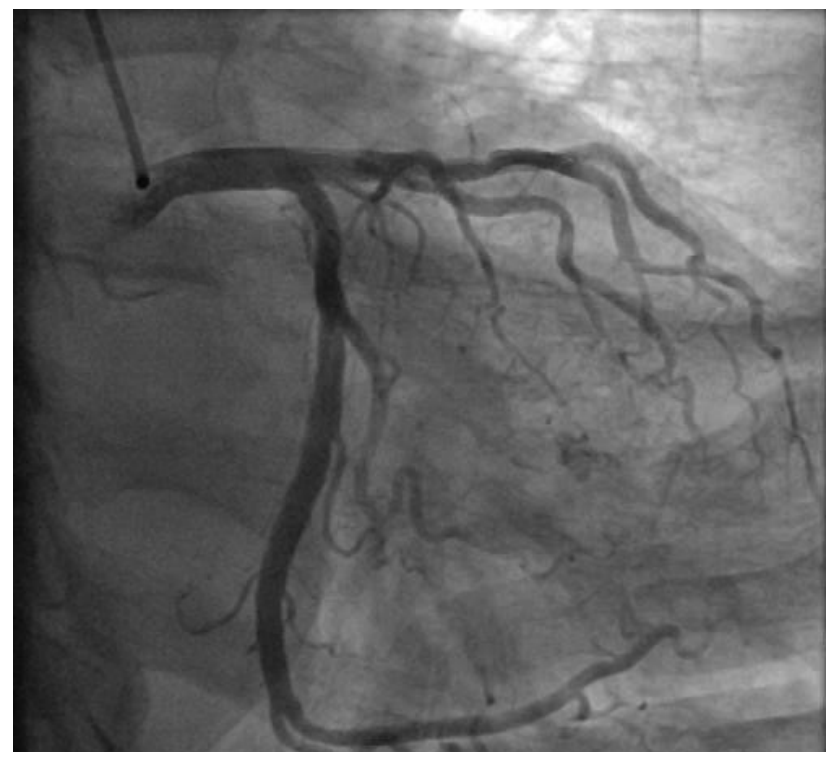

Figure 2. Coronary angiography after 2 weeks showed no residual obstructive lesions in the LAD and the LCX with TIMI 3 flow.

Despite that emergency thrombo-aspiration as well as balloon angioplasty was performed, the coronary blood flow kept TIMI (thrombolysis in myocardial infarction) 0 flow. Still, there appeared no improvement in the blood flow after intracoronary injection of 600 ug tirofiban. In consideration of her past history of untreated AF together with the angiography finding, AF-related coronary artery embolus was highly suggestive as the underlying pathophysiological evidence of the STEMI. $40 \mathrm{mg}$ recombinant human prourokinase was then administered via aspiration thrombectomy catheter.

Two weeks later, she underwent another coronary angiography showed that no residual obstructive lesions in the LAD and the LCX with TIMI 3 flow (Figure 2).

\section{Discussion}

AMI events are mainly the results of thrombotic occlusion of coronary arteries following rupture or erosion of atherosclerotic plaques. Nevertheless, CE is increasingly acknowledged as a vital non-atherosclerotic factor to induce AMI (3). In a prior report using coronary angiography or autopsy data, $4 \sim 7 \%$ of patients with AMI had no atherosclerotic coronary disease (5). Another study based on autopsy findings reported that coronary artery embolic infarct was found in $\sim 13 \%$ patients (55 out of 419) (6).

The current report on CE prevalence may be an underestimation, considering that elevated risks of thromboembolic incidence have been observed in patients with rheumatic valvular diseases, prosthesis, cardiomyopathy, intra-cardiac shunts, chronic AF, infective endocarditis, tumors, and several other hypercoagulable states $(7,8)$. As CE is less often, there is currently a lack of consensus guidelines for CE treatments. Among the possible therapeutic options, triple anti-platelet therapy 
(clopidogrel, IIb/IIIa receptor inhibitors, aspirin) (9), intracoronary catheter aspiration (10), PTCA (11), implantation of stents (12), as well as coronary arterial thrombectomy (13) have been shown to be successful.

However, in our case, despite of thrombus aspiration and balloon dilatation, as well as intracoronary injection of IIB/IIIA antagonist agent, were administered to the patient, there was no restore of blood flow of coronaries.

In the century that angioplasty still under-developed, thrombolytic treatment was the standard treatment for STEMI. However, it gets rarely practiced nowadays following the advance of primary percutaneous coronary intervention (PCI) technique. The most common cause of STEMI resulted from coronaries thrombosis is AF, and the most common origin of thrombus is the left appendage. The thrombus may be well organized. The pathophysiological components are different from the thrombus of unstable plaque rupture. Thrombus aspiration may do well in taking out the thrombus in order to restore blood flow, but not necessarily able to remove all the thrombus.

The standard treatment for cerebral thromboembolic event caused by AF is intravenous thrombolysis, and the successful rate is getting higher as the development of new lytic agent with less complication. Considered as a thromboembolic complication of AF, STEMI resulted by coronaries thromboembolism should receive thrombolytic treatment, and it might give a better outcome. Indeed, our patient was given alteplase lytic treatment after failed PCI, and repeated coronary angiography also showed resolution of clot. In retrospectively reconsideration, the thrombolytic treatment effect might be better if the drug was given via intracoronary route while intervention is ongoing.

\section{Conclusion}

In summary, CE-associated STEMI in AF patients is indeed a rare situation. Early systemic anti-thrombosis therapy as well as patient compliance are required to alleviate both short- and long-term adverse effects. Nonetheless, novel approaches for more effective CE treatments in the future are warranted.

\section{Conflict of interest}

The authors declare that they have no conflicts of interest to disclose.

\section{References}

1. Thygesen K, Alpert JS, Jaffe AS, Simoons ML, Chaitman $\mathrm{BR}$, White HD, et al. Third universal definition of myocardial infarction. Eur Heart J. 2012;33(20):2551-67.

2. Thygesen K, Task Force M, Alpert JS, Task Force M, White $H D$, Task Force M, et al. Universal definition of myocardial infarctionKristian Thygesen, Joseph S. Alpert and Harvey D. White on behalf of the Joint ESC/ACCF/AHA/WHF Task Force for the Redefinition of Myocardial Infarction. Eur Heart J. 2007;28(20):2525-38.

3. Shibata T, Kawakami S, Noguchi T, Tanaka T, Asaumi Y, Kanaya $T$, et al. Prevalence, clinical features, and prognosis of acute myocardial infarction attributable to coronary artery embolism. Circulation. 2015;132(4):241-50.

4. Chugh SS, Blackshear JL, Shen W-K, Hammill SC, Gersh BJ. Epidemiology and natural history of atrial fibrillation: clinical implications. J Am Coll Cardiol. 2001;37(2):371-8.

5. Waller BF. Atherosclerotic and nonatherosclerotic coronary artery factors in acute myocardial infarction. Cardiovasc Clin. 1989;20(1):29-104.

6. Prizel K, Hutchins GM, Bulkley BH. Coronary artery embolism and myocardial infarction: A clinicopathologic study of 55 patients. Ann Intern Med. 1978;88(2):155-61.

7. Park HS, Park JH, Jeong JO. Intracoronary catheter aspiration can be an adequate option in patients with acute myocardial infarction caused by left atrial myxoma. J Cardiovasc Ultrasound. 2009;17(4):145-7.

8. Charles RG, Epstein EJ, Holt S, Coulshed N. Coronary embolism in valvular heart disease. Q J Med. 1982;51(202):147-61.

9. Box LC, Hanak V, Arciniegas JG. Dual coronary emboli in peripartum cardiomyopathy. Tex Heart Inst $\mathrm{J}$. 2004;31(4):442-4.

10. Kiernan TJ, Flynn AM, Kearney P. Coronary embolism causing myocardial infarction in a patient with mechanical aortic valve prosthesis. Int J Cardiol. 2006;112(2):e14-6.

11. Hernandez F, Pombo M, Dalmau R, Andreu J, Alonso M, Albarran A, et al. Acute coronary embolism: angiographic diagnosis and treatment with primary angioplasty. Catheter Cardiovasc Interv. 2002;55(4):491-4.

12. Rifaie $\mathrm{O}$, Nammas W. Coronary air embolism during mitral valvuloplasty. Acta Cardiol. 2011;66(5):665-7.

13. Braun S, Schrotter H, Reynen K, Schwencke C, Strasser $\mathrm{RH}$. Myocardial infarction as complication of left atrial myxoma. Int J Cardiol. 2005;101(1):115-21. 